\title{
Predictive scoring models for persistent gram-negative bacteremia that reduce the need for follow-up blood cultures: a retrospective observational cohort study
}

Jongtak Jung ${ }^{1,2}$, Kyoung-Ho Song ${ }^{1,2^{*}}$ D , Kang II. Jun², Chang Kyoung Kang ${ }^{2}$, Nak-Hyun Kim,2, Pyoeng Gyun Choe ${ }^{2}$, Wan Beom Park ${ }^{2}$, Ji Hwan Bang ${ }^{2}$, Eu Suk Kim,2, Sang-Won Park ${ }^{2}$, Nam Joong Kim², Myoung-don Oh² and Hong Bin Kim ${ }^{1,2}$

\begin{abstract}
Background: Although the risk factors for positive follow-up blood cultures (FUBCs) in gram-negative bacteremia (GNB) have not been investigated extensively, FUBC has been routinely carried out in many acute care hospitals. We attempted to identify the risk factors and develop a predictive scoring model for positive FUBC in GNB cases.

Methods: All adults with GNB in a tertiary care hospital were retrospectively identified during a 2-year period, and GNB cases were assigned to eradicable and non-eradicable groups based on whether removal of the source of infection was possible. We performed multivariate logistic analyses to identify risk factors for positive FUBC and built predictive scoring models accordingly.

Results: Out of 1473 GNB cases, FUBCs were carried out in 1268 cases, and the results were positive in 122 cases. In case of eradicable source of infection, we assigned points according to the coefficients from the multivariate logistic regression analysis: Extended spectrum beta-lactamase-producing microorganism (+ 1 point), catheter-related bloodstream infection $(+1)$, unfavorable treatment response $(+1)$, quick sequential organ failure assessment score of 2 points or more $(+1)$, administration of effective antibiotics $(-1)$, and adequate source control $(-2)$. In case of non-eradicable source of infection, the assigned points were end-stage renal disease on hemodialysis $(+1)$, unfavorable treatment response $(+1)$, and the administration of effective antibiotics $(-2)$. The areas under the curves were 0.861 (95\% confidence interval [95Cl] $0.806-0.916)$ and $0.792(95 \mathrm{Cl}, 0.724-0.861)$, respectively. When we applied a cut-off of 0 , the specificities and negative predictive values (NPVs) in the eradicable and non-eradicable sources of infection groups were $95.6 / 92.6 \%$ and 95.5/ 95.0\%, respectively.

(Continued on next page)
\end{abstract}

\footnotetext{
* Correspondence: khsongmd@gmail.com; khsongmd@snu.ac.kr

${ }^{1}$ Department of Internal Medicine, Seoul National University Bundang

Hospital, 82, Gumi-ro173 Beon-gil, Bundang-gu, Seongnam-si, Gyeonggi-do

13620, Republic of Korea

${ }^{2}$ Department of Internal Medicine, Seoul National University College of

Medicine, Seoul, Republic of Korea
}

(c) The Author(s). 2020 Open Access This article is licensed under a Creative Commons Attribution 4.0 International License, which permits use, sharing, adaptation, distribution and reproduction in any medium or format, as long as you give appropriate credit to the original author(s) and the source, provide a link to the Creative Commons licence, and indicate if changes were made. The images or other third party material in this article are included in the article's Creative Commons licence, unless indicated otherwise in a credit line to the material. If material is not included in the article's Creative Commons licence and your intended use is not permitted by statutory regulation or exceeds the permitted use, you will need to obtain permission directly from the copyright holder. To view a copy of this licence, visit http://creativecommons.org/licenses/by/4.0/ The Creative Commons Public Domain Dedication waiver (http://creativecommons.org/publicdomain/zero/1.0/) applies to the data made available in this article, unless otherwise stated in a credit line to the data. 
(Continued from previous page)

Conclusions: FUBC is commonly carried out in GNB cases, but the rate of positive results is less than 10\%. In our simple predictive scoring model, zero scores - which were easily achieved following the administration of effective antibiotics and/or adequate source control in both groups — had high NPVs. We expect that the model reported herein will reduce the necessity for FUBCs in GNB cases.

Keywords: Gram-negative bacteremia, Follow-up blood culture, Persistent bacteremia, Risk factor, Predictive scoring model

\section{Background}

Although the positive rate of detection from follow-up blood cultures (FUBCs) in gram-negative bacteremia (GNB) is relatively low (5.8-10.9\%) [1-3], and the risk factors for persistent GNB have not been investigated extensively, FUBCs have been routinely conducted in cases of GNB in many acute care hospitals [1-4]. Unnecessary routine blood cultures are invasive, and false positives due to contamination increase medical costs and time spent in hospitals $[2,5,6]$. The authors of a previous study identified the risk factors for persistent bacteremia, and fever was found to be the only risk factor associated with GNB [1]. Owing to the rarity of persistent GNB, the previous study had limitations, including an insufficient number of persistent GNB cases.

Recently, several studies have reported that a shorter course of antibiotics in uncomplicated GNB (hemodynamically stable patients who have received effective antibiotics and adequate source control) $[7,8]$ does not produce an inferior prognosis compared to a longer course. Further, a recent randomized control study showed that a 7-day course of antibiotic therapy in uncomplicated GNB was not inferior to a 14-day course. Thus, FUBCs may not be necessary for the management of uncomplicated GNB, since it can be adequately treated by a short course of antibiotics [8].

Therefore, we attempted to identify the risk factors for a positive FUBC result in GNB and developed a predictive scoring model to reduce the need for performing unnecessary FUBCs.

\section{Methods \\ Patients}

We retrospectively reviewed all gram-negative episodes of bacteremia in a tertiary care university-affiliated 1300bed hospital in South Korea from December 1, 2015 to December 1, 2017. Patients under 18 years of age, those who died within 2 days, and patients with concomitant gram-positive bacteremia or fungemia were excluded from the study (Fig. 1). New episodes of bacteremia identified by FUBC (different species were identified by the FUBC than those identified in the initial blood culture) were also excluded when we compared the FUBCpositive and -negative groups. The study was approved by the institutional review board of Seoul National University Bundang Hospital.

\section{Variables and groups}

The variables were as follows: comorbidities, primary sources of infection, antibiotic status at the time of FUBC, identified microorganisms, susceptibility to antibiotics, fever, complete blood count, levels of C-reactive protein (CRP), quick sequential organ failure assessment (qSOFA) score, sequential organ failure assessment (SOFA) score, inotropic requirement, and source control status.

Patients who were subjected to FUBC 2-7 days after the initial blood culture, were assigned to the FUBC group, whereas patients who were not subjected to FUBC were assigned to the No-FUBC group. Cases were assigned to the FUBC-positive group if the same species of microorganism as the initial bacteremia was identified in at least one pair of FUBCs, and it was defined as persistent bacteremia. Cases in which bacteremia had been eliminated were assigned to the FUBC-negative group.

GNB was classified into eradicable and non-eradicable sources of infection according to the possibility of removal of the primary source of infection [9-11]; for example, removal of a central venous catheter or other endovascular devices; drainage of a dilated bile duct or hydronephrosis; surgical debridement of the skin, soft tissue infection, or osteomyelitis; drainage or removal of an intra-abdominal abscess; and drainage of empyema or a lung abscess.

An unfavorable treatment response was defined as the presence of at least two of the following variables: fever, aggravated leukocytosis, and no decrease in the level of CRP on the day that the FUBC was performed. Susceptible antibiotics were deemed to have been administered effectively when they were administered at least 1 calendar day before the FUBC according to the results of the antibiotics sensitivity test conducted in compliance with the Clinical and Laboratory Standards Institute (CLSI) guidelines. Adequate source control was defined as control of an eradicable source of infection at least 1 calendar day before the FUBC was performed [3, 10].

We compared the differences in clinical characteristics between the FUBC and the no-FUBC groups to assess 


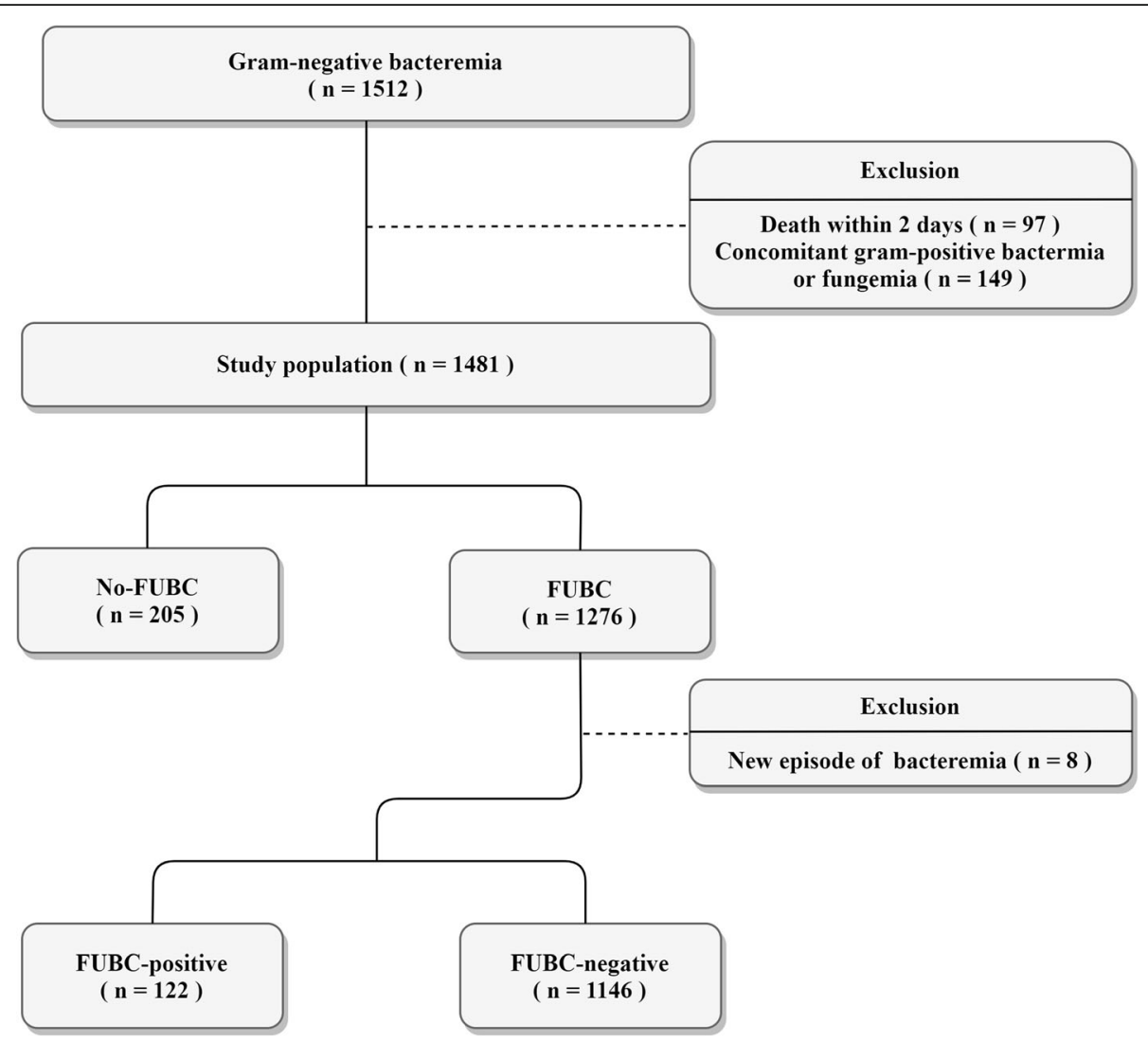

Fig. 1 Flow chart of the study. Inclusion and exclusion criteria for the study population. FUBC: follow-up blood culture

selection bias. Subsequently, we divided the FUBC group into eradicable and non-eradicable sources of infection and performed multivariate logistic regression analyses to identify the independent risk factors for a positive FUBC. If species different from the one causing the initial bacteremia were identified in the FUBC, it was considered a new bacteremia and excluded from the analysis. We built a predictive scoring model by assigning scores according to the beta-coefficient of the logistic regression analyses and verified it using receiver operating characteristic curve (ROC) analysis.

\section{Statistical analyses}

The statistical analyses were performed using IBM SPSS version 25.0. Fisher's exact and Chi-square tests were used to analyze categorical variables, and student's t-tests were used for continuous variables. Multivariate logistic regression analyses were performed using variables with $p$-values of less than 0.1 in the univariate analyses. A $p$-value of less than 0.05 was considered statistically significant in the multivariate analyses.

\section{Results}

Overall, 1481 GNB cases were identified during the study period. Of these, FUBCs were performed in 1276 cases (86.2\%), and 122 produced positive results (9.6\%) (Fig. 1). There were no cases of suspected contamination, and a new bacteremia was identified in eight cases of FUBC (Supplementary Table 1). Comparisons between the FUBC group and the no-FUBC group are shown in Table 1. Variables such as hematologic malignancy, the presence of an intravascular device, and the presence of extended spectrum beta lactamase (ESBL)-producing microorganisms were significantly higher in the FUBC group. There was no significant difference in the incidence of in-hospital mortality between the two groups.

Positive and negative clinical characteristics in the FUBC groups are compared in Table 2, according to the eradicability of the infection source, and the identified independent risk factors are listed in Table 3. In cases where the source of infection was eradicable, the independent risk factors were as follows: the presence of ESBLproducing microorganisms, catheter-related bloodstream infection, unfavorable treatment responses, a qSOFA score of at least 2 points, the administration of effective 
Table 1 Comparisons of clinical characteristics of gram-negative bacteraemia between the follow-up blood culture (FUBC) and noFUBC groups

\begin{tabular}{|c|c|c|c|}
\hline & FUBC-drawn $(n=1276)$ & No FUBC-drawn $(n=205)$ & $p$-value \\
\hline Age, years $( \pm S D)$ & $68.85( \pm 14.27)$ & $69.2( \pm 14.13)$ & 0.744 \\
\hline Sex (M) & $628(49.2 \%)$ & 117 (57.1\%) & $0.042^{*}$ \\
\hline Body weight (kg) & $59.90( \pm 11.56)$ & $59.24( \pm 12.62)$ & 0.533 \\
\hline \multicolumn{4}{|l|}{ Comorbidity } \\
\hline DM & $404(31.7 \%)$ & $70(34.1 \%)$ & 0.519 \\
\hline Liver cirrhosis & $95(7.4 \%)$ & $17(8.3 \%)$ & 0.776 \\
\hline ESRD on $\mathrm{HD}$ & $2(1.0 \%)$ & $35(2.7 \%)$ & 0.154 \\
\hline ESRD on PD & $6(0.5 \%)$ & $0(0.0 \%)$ & 1.000 \\
\hline Rheumatic disease & $18(1.4 \%)$ & $1(0.5 \%)$ & 0.500 \\
\hline Haematologic malignancy & $136(10.7 \%)$ & $7(3.4 \%)$ & $0.001^{*}$ \\
\hline Solid malignancy & $361(28.4 \%)$ & 60 (29.4\%) & 0.802 \\
\hline Solid organ transplantation & $34(2.7 \%)$ & $5(2.4 \%)$ & 1.000 \\
\hline Bone marrow transplantation & $28(2.2 \%)$ & $1(0.5 \%)$ & 0.167 \\
\hline Intravascular device & $308(24.1 \%)$ & 19 (9.3\%) & $0.000^{*}$ \\
\hline Neutropenia & $127(10.0 \%)$ & $6(2.9 \%)$ & $0.002^{*}$ \\
\hline High-dose steroid & $24(1.9 \%)$ & $1(0.5 \%)$ & 0.239 \\
\hline \multicolumn{4}{|l|}{ Microorganism } \\
\hline Escherichia coli & $722(56.6 \%)$ & $113(55.1 \%)$ & 0.705 \\
\hline Klebsiella pneumoniae & $250(19.6 \%)$ & $45(22.0 \%)$ & 0.451 \\
\hline Pseudomonas aeruginosa & $62(4.9 \%)$ & 7 (3.4\%) & 0.384 \\
\hline AmpC-encoded Enterobacteriaceae ${ }^{a}$ & $100(7.8 \%)$ & $21(10.2 \%)$ & 0.237 \\
\hline Acinetobacter baumannii & $24(1.9 \%)$ & $1(0.5 \%)$ & 0.239 \\
\hline ESBL-producing & $313(24.5 \%)$ & $32(15.6 \%)$ & $0.006^{*}$ \\
\hline Other gram-negative & $110(8.6 \%)$ & $17(8.3 \%)$ & 0.895 \\
\hline PBSI & $50(3.9 \%)$ & $8(3.9 \%)$ & 1.000 \\
\hline Hospital onset & $328(25.7 \%)$ & $53(25.9 \%)$ & 1.000 \\
\hline \multicolumn{4}{|l|}{ Site of infection } \\
\hline Urinary genital tract & $502(39.3 \%)$ & $71(34.6 \%)$ & 0.217 \\
\hline Liver abscess & $56(4.4 \%)$ & $7(3.4 \%)$ & 0.583 \\
\hline Biliary & $303(23.7 \%)$ & $86(42.0 \%)$ & $0.000^{*}$ \\
\hline Intra-abdominal & $135(10.6 \%)$ & $18(8.8 \%)$ & 0.462 \\
\hline Respiratory & $56(4.4 \%)$ & $5(2.4 \%)$ & 0.255 \\
\hline SSTI & $13(1.0 \%)$ & $1(0.5 \%)$ & 0.707 \\
\hline Catheter-related & $40(3.1 \%)$ & $2(1.0 \%)$ & 0.109 \\
\hline Bone and joint infection & $12(0.9 \%)$ & $1(0.5 \%)$ & 1.000 \\
\hline Cardiovascular & $3(0.2 \%)$ & $0(0.0 \%)$ & 1.000 \\
\hline CNS infection & $3(0.2 \%)$ & $0(0.0 \%)$ & 1.000 \\
\hline Primary bacteraemia & $143(11.2 \%)$ & $10(4.9 \%)$ & $0.006^{*}$ \\
\hline In-hospital mortality & $87(6.8 \%)$ & $18(8.8 \%)$ & 0.379 \\
\hline
\end{tabular}

SD Standard deviation, $M$ Male, DM Diabetes mellitus, ESRD End-stage renal disease, HD Haemodialysis, PD Peritoneal dialysis, ESBL Extended-spectrum betalactamase, PBSI Polymicrobial bloodstream infection, SSTI Skin and soft tissue infection, CNS Central nervous system

${ }^{a} \mathrm{AmpC}$-encoded Enterobacteriaceae includes Serratia marcescens, Providencia stuartii, Proteus vulgaris, Citrobacter spp., Enterobacter spp., and Morganella morganii ${ }^{*} p<0.05$ 
Table 2 Clinical characteristics of gram-negative bacteraemia according to the results of follow-up blood culture (FUBC) and the eradicability of the source of infection

\begin{tabular}{|c|c|c|c|c|c|c|}
\hline & \multicolumn{3}{|c|}{ Eradicable source of infection } & \multicolumn{3}{|c|}{ Non-eradicable source of infection } \\
\hline & $\begin{array}{l}\text { Positive FUBC } \\
(n=55)\end{array}$ & $\begin{array}{l}\text { Negative FUBC }(n= \\
411)\end{array}$ & $\begin{array}{l}p \text { - } \\
\text { value }\end{array}$ & $\begin{array}{l}\text { Positive FUBC } \\
(n=66)\end{array}$ & $\begin{array}{l}\text { Negative FUBC }(n= \\
736)\end{array}$ & $\begin{array}{l}p \text { - } \\
\text { value }\end{array}$ \\
\hline Age, years $( \pm S D)$ & $69.18( \pm 12.19)$ & $70.15( \pm 13.35)$ & 0.610 & $70.15( \pm 14.25)$ & $67.98( \pm 14.88)$ & 0.254 \\
\hline Sex (M) & $29(52.7 \%)$ & $222(54.0 \%)$ & 0.886 & $26(39.4 \%)$ & $346(47.0 \%)$ & 0.235 \\
\hline \multicolumn{7}{|l|}{ Body weight (kg) } \\
\hline \multicolumn{7}{|l|}{ Comorbidity } \\
\hline DM & 19 (34.5\%) & $121(29.4 \%)$ & 0.531 & $25(37.9 \%)$ & $238(32.3 \%)$ & 0.358 \\
\hline Liver cirrhosis & $6(10.9 \%)$ & $31(7.5 \%)$ & 0.422 & $4(6.1 \%)$ & $54(7.3 \%)$ & 1.000 \\
\hline ESRD on HD & $4(7.3 \%)$ & $7(1.7 \%)$ & $0.031^{*}$ & $6(9.1 \%)$ & $18(2.4 \%)$ & $0.010^{*}$ \\
\hline ESRD on PD & $0(0.0 \%)$ & $0(0.0 \%)$ & N.A. & $2(3.0 \%)$ & $4(.0 .5 \%)$ & 0.081 \\
\hline Rheumatic disease & $1(1.8 \%)$ & $0(0.0 \%)$ & 0.119 & $2(3.0 \%)$ & $15(2.0 \%)$ & 0.644 \\
\hline Haematologic malignancy & $4(7.3 \%)$ & $8(2.0 \%)$ & $0.042^{*}$ & $12(18.2 \%)$ & $109(14.9 \%)$ & 0.474 \\
\hline Solid malignancy & $17(30.9 \%)$ & $149(36.4 \%)$ & 0.457 & $16(24.2 \%)$ & $176(24.0 \%)$ & 0.966 \\
\hline Solid organ transplantation & $2(3.6 \%)$ & $9(2.2 \%)$ & 0.627 & $4(6.1 \%)$ & $19(2.6 \%)$ & 0.113 \\
\hline Bone marrow transplantation & $2(3.6 \%)$ & $1(0.2 \%)$ & $0.038^{*}$ & $2(3.0 \%)$ & $22(3.0 \%)$ & 1.000 \\
\hline Intravascular device & $75(18.2 \%)$ & $26(47.3 \%)$ & $0.000^{*}$ & $4(6.1 \%)$ & $19(2.6 \%)$ & 0.113 \\
\hline Neutropenia & $1(1.8 \%)$ & $8(2.0 \%)$ & 1.000 & $11(16.7 \%)$ & $105(14.3 \%)$ & 0.608 \\
\hline High-dose steroid & $1(1.8 \%)$ & $4(1.0 \%)$ & 0.468 & $4(6.1 \%)$ & $15(2.0 \%)$ & 0.063 \\
\hline \multicolumn{7}{|l|}{ Microorganism } \\
\hline Escherichia coli & $22(40.0 \%)$ & 205 (49.9\%) & 0.197 & $36(54.5 \%)$ & $456(62.0 \%)$ & 0.291 \\
\hline Klebsiella pneumoniae & $11(20.0 \%)$ & $91(22.1 \%)$ & 0.735 & $14(21.2 \%)$ & $133(18.1 \%)$ & 0.527 \\
\hline Pseudomonas aeruginosa & $5(9.1 \%)$ & $19(4.6 \%)$ & 0.185 & $5(7.6 \%)$ & $33(4.5 \%)$ & 0.231 \\
\hline vAmpC-encoded Enterobacteriaceae ${ }^{a}$ & $7(12.7 \%)$ & 49 (11.9\%) & 1.000 & $4(6.1 \%)$ & $39(5.3 \%)$ & 0.774 \\
\hline Acinetobacter baumannii & $1(1.8 \%)$ & $6(1.5 \%)$ & 0.587 & $2(3.0 \%)$ & $15(2.0 \%)$ & 0.644 \\
\hline ESBL-producing & $21(38.2 \%)$ & $84(20.4 \%)$ & $0.003^{*}$ & $39(59.1 \%)$ & $169(23.0 \%)$ & $0.000^{*}$ \\
\hline Other gram-negative & $9(16.4 \%)$ & $34(8.3 \%)$ & $0.052^{*}$ & $5(7.6 \%)$ & $59(8.0 \%)$ & 0.899 \\
\hline PBSI & $5(9.1 \%)$ & $32(7.8 \%)$ & 0.789 & $1(1.5 \%)$ & $12(1.6 \%)$ & 1.000 \\
\hline Hospital onset & $22(40.0 \%)$ & $66(16.1 \%)$ & $0.000^{*}$ & 19 (28.8\%) & $216(29.3 \%)$ & 1.000 \\
\hline \multicolumn{7}{|l|}{ Site of infection } \\
\hline Urinary genital tract & $12(21.8 \%)$ & $80(19.5 \%)$ & 0.718 & $38(57.6 \%)$ & $372(50.5 \%)$ & 0.305 \\
\hline Liver abscess & $2(3.6 \%)$ & $39(9.5 \%)$ & 0.205 & $0(0.0 \%)$ & $13(1.8 \%)$ & 0.615 \\
\hline Biliary infection & $9(16.4 \%)$ & $218(53.0 \%)$ & $0.000^{*}$ & $1(1.5 \%)$ & $73(9.9 \%)$ & $0.024^{*}$ \\
\hline Intra-abdominal & $5(9.1 \%)$ & $38(9.2 \%)$ & 1.000 & $6(9.1 \%)$ & $85(11.5 \%)$ & 0.687 \\
\hline Respiratory & $1(1.8 \%)$ & $2(0.5 \%)$ & 0.315 & $7(10.6 \%)$ & $44(6.0 \%)$ & 0.180 \\
\hline SSTI & $3(5.5 \%)$ & $4(1.0 \%)$ & $0.039^{*}$ & $2(3.0 \%)$ & $4(0.5 \%)$ & 0.081 \\
\hline Catheter-related & $18(32.1 \%)$ & $22(5.4 \%)$ & $0.000^{*}$ & $0(0.0 \%)$ & $0(0.0 \%)$ & N.A. \\
\hline Bone and joint infection & $3(5.5 \%)$ & $2(0.5 \%)$ & $0.013^{*}$ & $1(1.5 \%)$ & $6(0.8 \%)$ & 0.453 \\
\hline Cardiovascular & $2(3.6 \%)$ & $1(0.2 \%)$ & $0.038^{*}$ & $0(0.0 \%)$ & $0(0.0 \%)$ & N.A. \\
\hline CNS infection & $0(0.0 \%)$ & $0(0.0 \%)$ & N.A. & $0(0.0 \%)$ & $2(0.2 \%)$ & 1.000 \\
\hline Primary bacteraemia & $0(0.0 \%)$ & $0(0.0 \%)$ & N.A. & $11(16.7 \%)$ & $131(17.8 \%)$ & 0.869 \\
\hline $\begin{array}{l}\text { Inotropic requirement on the day of } \\
\text { FUBC }\end{array}$ & $24(5.8 \%)$ & $10(18.2 \%)$ & $0.003^{*}$ & $9(13.6 \%)$ & $53(7.2 \%)$ & 0.061 \\
\hline Unfavourable treatment response & $30(55.6 \%)$ & $130(32.5 \%)$ & $0.001^{*}$ & $31(48.4 \%)$ & $215(30.0 \%)$ & $0.002^{*}$ \\
\hline qSOFA score $( \pm S D)$ on the day of FUBC & $1.29( \pm 1.12)$ & $0.52( \pm 0.76)$ & $0.000^{*}$ & $0.88( \pm 0.95)$ & $0.56( \pm 0.88)$ & $0.005^{*}$ \\
\hline
\end{tabular}


Table 2 Clinical characteristics of gram-negative bacteraemia according to the results of follow-up blood culture (FUBC) and the eradicability of the source of infection (Continued)

\begin{tabular}{|c|c|c|c|c|c|c|}
\hline & \multicolumn{3}{|c|}{ Eradicable source of infection } & \multicolumn{3}{|c|}{ Non-eradicable source of infection } \\
\hline & $\begin{array}{l}\text { Positive FUBC } \\
(n=55)\end{array}$ & $\begin{array}{l}\text { Negative FUBC }(n= \\
411)\end{array}$ & $\begin{array}{l}p \text { - } \\
\text { value }\end{array}$ & $\begin{array}{l}\text { Positive FUBC } \\
(n=66)\end{array}$ & $\begin{array}{l}\text { Negative FUBC }(n= \\
\text { 736) }\end{array}$ & $\begin{array}{l}p \text { - } \\
\text { value }\end{array}$ \\
\hline SOFA score ( \pm SD) on the day of FUBC & $5.62( \pm 4.48)$ & $3.09( \pm 2.93)$ & $0.000^{*}$ & $3.79( \pm 3.47)$ & $2.90( \pm 3.18)$ & $0.036^{*}$ \\
\hline qSOFA score $\geq 2$ on the day of FUBC & $20(36.4 \%)$ & $45(10.9 \%)$ & $0.000^{*}$ & $16(24.2 \%)$ & $108(14.7 \%)$ & 0.039 \\
\hline $\begin{array}{l}\text { Effective antibiotics before the day of } \\
\text { FUBC }\end{array}$ & $34(61.8 \%)$ & $350(85.2 \%)$ & $0.000^{*}$ & $27(40.9 \%)$ & $639(86.8 \%)$ & $0.000^{*}$ \\
\hline $\begin{array}{l}\text { Adequate source control before the day } \\
\text { of FUBC }\end{array}$ & $13(23.6 \%)$ & $322(78.3 \%)$ & $0.000^{*}$ & N.A. & N.A. & N.A. \\
\hline
\end{tabular}

SD Standard deviation, N.A. Not available, M Male, DM Diabetes mellitus, ESRD End-stage renal disease, HD Haemodialysis, PD Peritoneal dialysis, ESBL Extendedspectrum beta-lactamase, PBSI Polymicrobial bloodstream infection, SSTI Skin and soft tissue infection, CNS Central nervous system

${ }^{a} \mathrm{AmpC}$-encoded Enterobacteriaceae includes Serratia marcescens, Providencia stuartii, Proteus vulgaris, Citrobacter spp., Enterobacter spp., and Morganella morganii ${ }^{*} p<0.05$

antibiotics, and adequate source control. In cases where the source of infection was non-eradicable, the independent risk factors were as follows: end-stage renal disease on hemodialysis, unfavorable treatment responses, and the administration of effective antibiotics. We assigned points to the independent risk factors for positive FUBC, based on the beta-coefficients from the logistic regression analysis (Table 3). In cases of eradicable and non-eradicable sources of infection, the values of the area under the curve of the receiver operating characteristic curve (AUC-ROC) of each scoring model were 0.861 (95\% confidence interval (CI) $0.806-0.916)$ and 0.792 (95\% CI, 0.724-0.861), respectively (Table 3, Fig. 2). The sensitivities, specificities, positive predictive values (PPVs), and negative predictive values (NPVs) according to the cut-off values are listed in Table 4.

When we applied a cut-off value of 0 , the specificities of the eradicable and non-eradicable sources of infection were 95.6 and $95.5 \%$, respectively. The NPVs of the predictive scoring models in the eradicable and noneradicable source infections were 92.6 and $95.0 \%$, respectively.

The percentage of positive FUBCs according to the scores of eradicable and non-eradicable source of infection is shown in Fig. 3. The percentage of positive FUBCs was less than $2 \%$ in cases with -3 or -2 points of eradicable or non-eradicable sources of infection, but 40 and $70 \%$ had positive FUBCs in cases with 1 or 2

Table 3 Independent risk factors and assigned scores used to build the predictive scoring model for positive follow-up blood culture in gram-negative bacteraemia, according to eradicable and non-eradicable sources of infection

\begin{tabular}{|c|c|c|c|c|}
\hline \multicolumn{5}{|l|}{ Eradicable source of infection } \\
\hline & Beta-coefficient & Odds ratio $(95 \% \mathrm{Cl})$ & $p$-value & Assigned score \\
\hline ESBL-producing microorganism infection & 1.001 & $2.720(1.179-6.271)$ & 0.019 & +1 \\
\hline CRBSI & 1.374 & $3.95(1.522-10.255)$ & 0.005 & +1 \\
\hline Unfavourable treatment response $\mathrm{a}^{\mathrm{a}}$ & 0.802 & $2.229(1.262-3.937)$ & 0.006 & +1 \\
\hline qSOFA score $\geq 2$ on the day of FUBC & 0.864 & $2.371(1.034-5.438)$ & 0.041 & +1 \\
\hline Effective antibiotics administration before the day of FUBC & -1.007 & $0.365(0.164-0.814)$ & 0.014 & -1 \\
\hline Adequate source control before the day of FUBC & -1.983 & $0.138(0.064-0.294)$ & 0.000 & -2 \\
\hline \multicolumn{5}{|l|}{ Non-eradicable source of infection } \\
\hline ESRD on HD & 1.406 & $4.081(1.331-12.515)$ & 0.014 & +1 \\
\hline Unfavourable treatment response $\mathrm{a}^{\mathrm{a}}$ & 0.802 & $2.229(1.262-3.937)$ & 0.006 & +1 \\
\hline Effective antibiotics administration before the day of FUBC & -2.015 & $0.133(0.069-0.258)$ & 0.000 & -2 \\
\hline
\end{tabular}

CI Confidence interval, ESBL Extended-spectrum beta-lactamase, CRBSI Catheter-related bloodstream infection, qSOFA Quick sequential organ failure assessment, FUBC Follow-up blood culture, ESRD End-stage renal disease, $H D$ Haemodialysis

a Unfavourable treatment response was defined as positivity for at least 2 variables among the presence of fever, aggravated leucocytosis, and no decrease of Creactive protein on the day of FUBC 


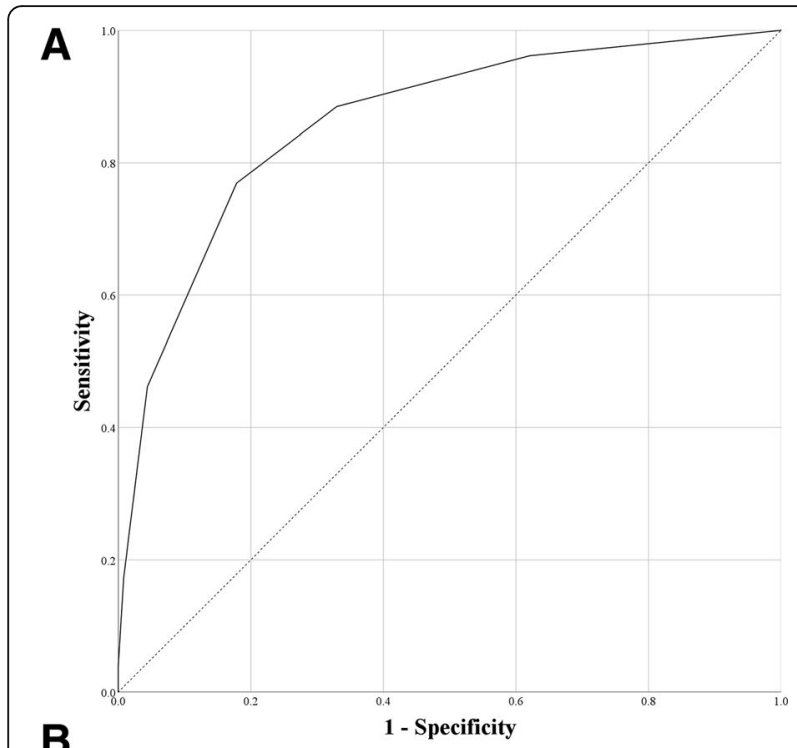

B

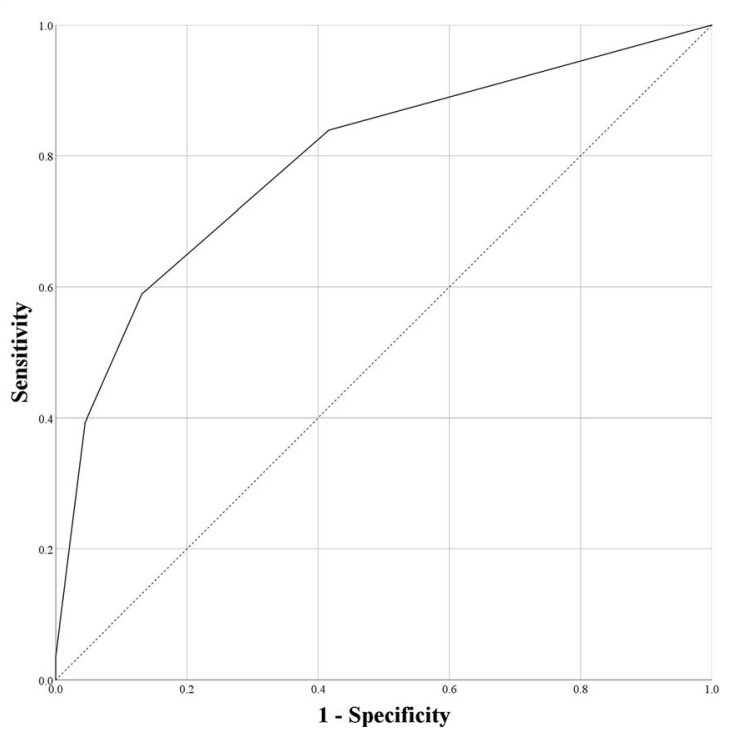

Fig. 2 a Receiver operating characteristic (ROC) analysis of the predictive scoring model in cases with eradicable sources of infection. The area under the curve (AUC) was 0.861 (95\% confidence interval (CI) $0.806-0.916)$. b ROC analysis of the predictive scoring model in cases with non-eradicable sources of infection. The AUC was $0.792(95 \% \mathrm{Cl}, 0.724-0.861)$

points of non-eradicable or eradicable sources of infection, respectively.

\section{Discussion}

Our study revealed that FUBCs were performed in most of the patients with GNB, but less than $10 \%$ produced positive results. In contrast to gram-positive bacteremia, positive FUBC results were not common in GNB $[1,3]$.
In eradicable sources of infection, variables such as ESBL-producing organisms, catheter-related bloodstream infections (CRBSIs), unfavorable treatment responses, and qSOFA scores of at least 2 were independent risk factors, but when effective antibiotics were administered and there was adequate source control the day before performing FUBC, the probability of negative conversion of bacteremia in FUBC increased significantly. When appropriate management (e.g., administration of effective antibiotics and source control) was performed, and there was a clear clinical response (qSOFA score $<2$ ), the score predicted by the model did not exceed the cut-off value (zero points), and the probability of negative conversion in FUBC was as high as 92.6\%. In Staphylococcus aureus infections, delays in the removal of eradicable sources of infection, the initial administration of inappropriate antibiotics, and delays in the delivery of appropriate antibiotics were important risk factors for persistent bacteremia [10, 12]. Early source control (within 48 h) was also important for eradicating bacteremia in both grampositive and gram-negative bacteremia [3]. Therefore, as with $S$. aureus bacteremia, in cases of GNB with eradicable sources of infection-regardless of the site of infection, underlying diseases, or causative microorganisms-if there is appropriate management and the clinical response is favorable, FUBC provides little benefit.

In cases in which the source of infection was noneradicable, hemodialysis and an unfavorable treatment response were independent risk factors. However, if effective antibiotics were administered to the patient, bacteremia was usually eliminated. If effective antibiotics were administered-regardless of the underlying disease, microorganism type, or treatment response-the score did not exceed the cut off-value (zero points), and the probability of negative conversion of bacteremia was 95.0\%.

If FUBC was performed only in cases with more than zero points of both eradicable and non-eradicable source of infection, more than $90 \%$ of FUBCs could have been avoided in our study population (Fig. 3). Even if the cutoff value was more strictly set as -1 to increase the NPV, more than $70 \%$ of FUBCs could have been saved. Therefore, performing FUBC to evaluate the response to treatment can be avoided in most uncomplicated cases of GNB. In GNB cases, FUBC could be applied selectively to patients with a high risk of positive FUBCs, unlike in S. aureus bacteremia or candidemia.

The contamination rate of peripheral blood cultures in our hospital is as low as $0.5 \%$, and among the 1276 cases of FUBC in our study, there were no suspected cases of contamination. However, contamination rates in blood cultures have been reported ranging from $0.9-7.9 \%$ [5]. In other studies related to FUBCs, contaminants were identified in $2.0-3.9 \%$ of FUBCs [13, 14]. Therefore, 
Table 4 Receiver operating characteristics and predictability of scoring models for positive follow-up blood culture in gram-negative bacteraemia, according to the various cut-off values

\begin{tabular}{|c|c|c|c|c|c|c|}
\hline & AUC (95\% Cl) & Cut-off & Sensitivity & Specificity & PPV & NPV \\
\hline \multirow[t]{5}{*}{ Eradicable source of infection $^{a}$} & $0.861(0.806-0.916)$ & -2 & $88.5 \%$ & $67.0 \%$ & $27.7 \%$ & $97.6 \%$ \\
\hline & & -1 & $76.9 \%$ & $82.1 \%$ & $38.1 \%$ & $96.1 \%$ \\
\hline & & 0 & $46.2 \%$ & $95.6 \%$ & $60.0 \%$ & $92.6 \%$ \\
\hline & & 1 & $17.3 \%$ & $99.3 \%$ & $75 \%$ & $90.63 \%$ \\
\hline & & 2 & $3.8 \%$ & $100 \%$ & $100 \%$ & $87.9 \%$ \\
\hline \multirow[t]{4}{*}{ Non-eradicable source of infection } & $0.792(0.724-0.861)$ & -2 & $83.9 \%$ & $58.4 \%$ & $14.4 \%$ & $97.6 \%$ \\
\hline & & -1 & $58.9 \%$ & $86.9 \%$ & $27.3 \%$ & $96.2 \%$ \\
\hline & & 0 & $39.3 \%$ & $95.5 \%$ & $42.3 \%$ & $95.0 \%$ \\
\hline & & 1 & $3.6 \%$ & $100.0 \%$ & $100.0 \%$ & $92.5 \%$ \\
\hline
\end{tabular}

AUC Area under the curve, Cl Confidence interval, PPV Positive predictive value, NPV Negative predictive value

${ }^{a}$ Gram-negative bacteraemia in which the primary source of infection could be removed, e.g. removal of a central venous catheter or other endovascular device, drainage of a dilated bile duct or hydronephrosis, surgical debridement of skin and soft tissue infection or osteomyelitis, drainage or removal of an intraabdominal abscess, or drainage of empyema or a lung abscess

universal FUBCs of all gram-negative bacteremia may produce contamination, which could lead to increased medical costs and prolonged hospital stays [5].

Recently, one study revealed that FUBC was a useful diagnostic tool for differentiating septic thrombophlebitis in gram-negative bacteremia of patients admitted to the intensive care unit (ICU) for polytrauma [15]. If the patient had a high-risk of persistent GNB and risk factors for deep vein thrombosis, such as the presence of a central catheter, multiple traumas or admission to the ICU $[16$, 17], further diagnostic evaluation, such as $C T$ angiography and duplex sonography as well as FUBCs to differentiate septic thrombophlebitis, could be considered.

Recent studies revealed that when FUBCs were performed in cases of gram-negative bloodstream infection, they were associated with improved clinical outcomes $[13,14]$. However, the proportion of FUBCs performed was relatively low (17.6-68\%) compared to our present study (86.2\%). In addition, the rates of positive FUBC $(20-38.5 \%)$ and all-cause mortality $(10-13.7 \%)$ were higher than in our study population $(9.7 \%$ positiveFUBC rate and $6.8 \%$ in-hospital mortality in the FUBC group). This means that FUBCs were selectively performed in severe GNB cases in these previous studies. It cannot be concluded that FUBC improves the prognosis in all GNB, especially in uncomplicated bacteremia. The FUBC group in these studies had a trend of longer treatment duration and hospital stays than the no-FUBC group, and contaminants were identified in $2.0-3.9 \%$ of FUBCs. If FUBC is conducted routinely in uncomplicated GNB, it may increase medical costs and cause various side effects due to prolonged antibiotic treatment. Therefore, the decision of conducting an FUBC should be made carefully, and our predictive scoring model will help with that decision. Appropriately conducted
FUBCs, according to the results of our predictive scoring model, may improve clinical outcomes and reduce side effects and medical costs.

Our study has some limitations. First, there may have been bias towards the FUBC group, because the study was conducted retrospectively. However, variables such as hematologic malignancy, presence of an intravascular device, and ESBL-producing microorganisms-which were significantly more prevalent in the FUBC group than in the no-FUBC group- were also significant risk factors for positive FUBCs (Table 1). This means that there was a higher probability of persistent bacteremia in the FUBC group than in the no-FUBC group. The NPVs of predictive scoring models in real-world cases of GNB would be higher than those indicated in the present study. Therefore, this selection bias did not alter our conclusion. Second, we did not determine how FUBC affects patient outcomes such as mortality, morbidity, length of stay in hospital, or total cost of medical care. Therefore, our findings alone will not be sufficient to change routine practice. As discussed above, recent studies have shown that FUBC was associated with improved outcome of GNB [13, 14], but these results couldn't be applied to the uncomplicated GNB. Further investigations such as a prospective randomized control study should be conducted to reveal the exact clinical impact of FUBC in GNB.

\section{Conclusions}

In this study, although FUBC was commonly used in cases of GNB, there were few positive results $(<10 \%$ of cases). We expect that the application of our simple predictive scoring model will reduce the need for performing unnecessary FUBC in uncomplicated cases of GNB. 


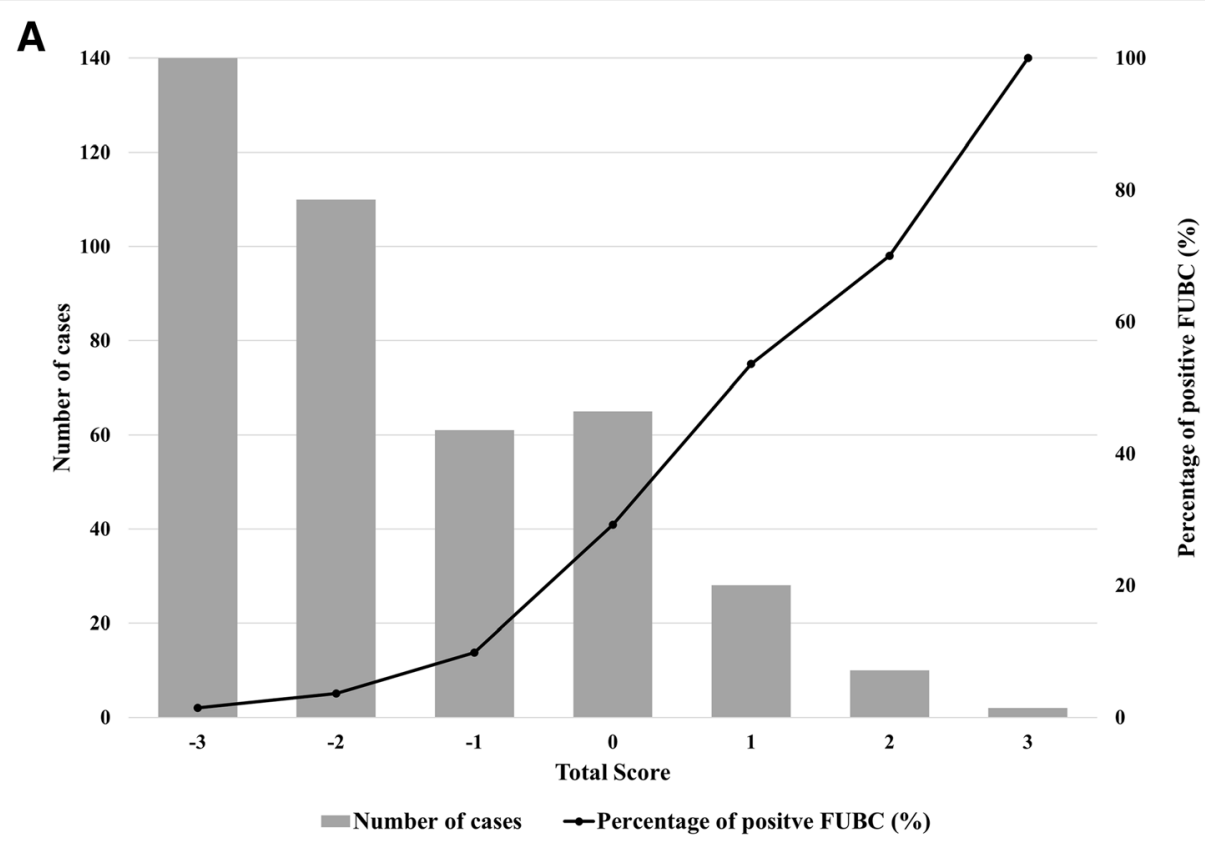

B

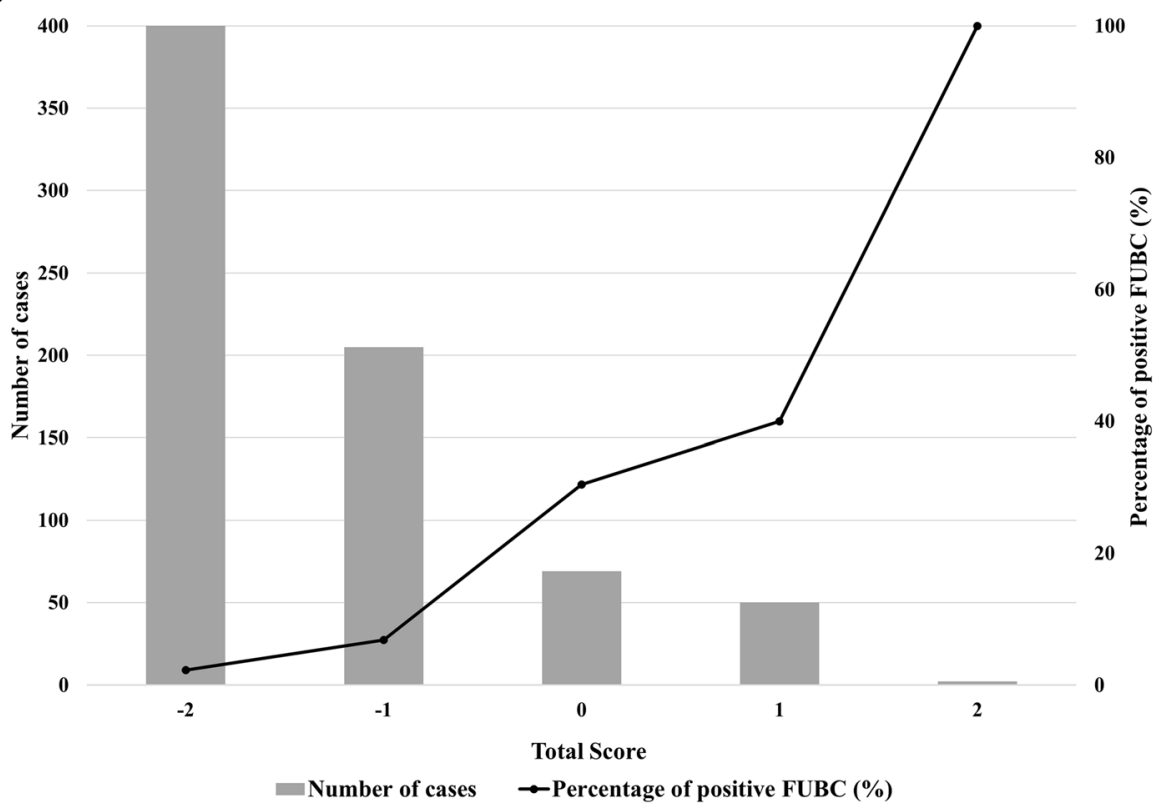

Fig. 3 a The percentage of positive follow-up blood cultures according to the scores of eradicable and $\mathbf{b}$ non-eradicable source of infection 


\section{Supplementary information}

Supplementary information accompanies this paper at https://doi.org/10. 1186/s12879-020-05395-8.

Additional file $\mathbf{1}$ Table $\mathbf{S 1}$. Summarized 8 cases of new bacteremia.

\section{Abbreviations}

FUBC: Follow-up blood culture; GNB: Gram-negative bacteremia; CRP: Creactive protein; qSOFA) score: Quick sequential organ failure assessment; ESBL: Extended spectrum beta lactamase; Cl: Confidence interval; PPVs: Positive predictive values; NPVs: Negative predictive values; CRBSI: Catheter-related bloodstream infection

\section{Acknowledgments}

We would like to thank Editage (www.editage.co.kr) for English language editing.

\section{Authors' contributions}

Conceptualization, Methodology, Writing - original draft, Investigation, Data curation, Formal analysis: J. J, K.H.S, Writing - review \& editing: K.I.J, C.K.K, N.H.K, P.G.C, J.H.B, Data interpretation, Writing - review \& editing: K.H.S, W.B.P, E.S.K, S.W.P, N.J.K, M.D.O, H.B.K. All authors have provided final approval for the final version of the manuscript.

\section{Funding}

This study did not receive any specific funding

\section{Availability of data and materials}

The datasets used and/or analyzed during the study are available from the corresponding author on reasonable request.

\section{Ethics approval and consent to participate}

The study was approved by the institutional review board of Seoul National University Bundang Hospital (B-1805/468-101). and the requirement for informed consent was waived due to the retrospective design of the study. The collected data was anonymized before its use.

\section{Consent for publication}

Not applicable.

\section{Competing interests}

There are no conflicts of interest to declare.

Received: 2 July 2020 Accepted: 3 September 2020

Published online: 17 September 2020

\section{References}

1. Canzoneri CN, Akhavan BJ, Tosur Z, Andrade PEA, Aisenberg GMA. Followup blood cultures in gram-negative bacteremia: are they needed? Clin Infect Dis. 2017;65(11):1776-9.

2. Kang CK, Kim ES, Song K-H, Kim HB, Kim TS, Kim N-H, Kim C-J, Choe PG, Bang J-H, Park WB. Can a routine follow-up blood culture be justified in Klebsiella pneumoniae bacteremia? A retrospective case-control study. BMC Infect Dis. 2013;13(1):365

3. Wiggers $J B$, Xiong $W$, Daneman $N$. Sending repeat cultures: is there a role in the management of bacteremic episodes? (SCRIBE study). BMC Infect Dis. 2016;16(1):286

4. Shi H, Kang Cl, Cho SY, Huh K, Chung DR, Peck KR. Follow-up blood cultures add little value in the management of bacteremic urinary tract infections. Eur J Clin Microbiol Infect Dis. 2019;38(4):695-702.

5. Dempsey C, Skoglund E, Muldrew KL, Garey KW. Economic health care costs of blood culture contamination: a systematic review. Am J Infect Control. 2019;47(8):963-7.

6. Doern GV, Carroll KC, Diekema DJ, Garey KW, Rupp ME, Weinstein MP, Sexton DJ. A comprehensive update on the problem of blood culture contamination and a discussion of methods for addressing the problem. Clin Mlcrobiol Rev. 2019;33(1):e00009-19.

7. Park SH, Milstone AM, Diener-West M, Nussenblatt V, Cosgrove SE, Tamma PD. Short versus prolonged courses of antibiotic therapy for children with uncomplicated gram-negative bacteraemia. J Antimicrob Chemother. 2013; 69(3):779-85.

8. Yahav D, Franceschini E, Koppel F, Turjeman A, Babich T, Bitterman R, Neuberger A, Ghanem-Zoubi N, Santoro A, Eliakim-Raz N. Seven versus 14 days of antibiotic therapy for uncomplicated gram-negative bacteremia: a noninferiority randomized controlled trial. Clin Infect Dis. 2019;69(7):1091-8.

9. Liu C, Bayer A, Cosgrove SE, Daum RS, Fridkin SK, Gorwitz RJ, Kaplan SL, Karchmer AW, Levine DP, Murray BE. Clinical practice guidelines by the Infectious Diseases Society of America for the treatment of methicillinresistant Staphylococcus aureus infections in adults and children. Clin Infect Dis. 2011;52(3):e18-55.

10. Chong YP, Park SJ, Kim HS, Kim ES, Kim MN, Park KH, Kim SH, Lee SO, Choi $\mathrm{SH}$, Jeong JY, et al. Persistent Staphylococcus aureus bacteremia: a prospective analysis of risk factors, outcomes, and microbiologic and genotypic characteristics of isolates. Medicine (Baltimore). 2013:92(2):98-108

11. Kim S-H, Park W-B, Lee K-D, Kang C-I, Kim H-B, Oh M-d, Kim E-C, Choe K-W. Outcome of Staphylococcus aureus bacteremia in patients with eradicable foci versus noneradicable foci. Clin Infect Dis. 2003;37(6):794-9.

12. Hawkins C, Huang J, Jin N, Noskin GA, Zembower TR, Bolon M. Persistent Staphylococcus aureus bacteremia: an analysis of risk factors and outcomes. Arch Intern Med. 2007;167(17):1861-7.

13. Giannella M, Pascale R, Pancaldi L, Monari C, lanniruberto S, Malosso P, Bussini L, Bartoletti M, Tedeschi S, Ambretti S, et al. Follow-up blood cultures are associated with improved outcome of patients with gramnegative bloodstream infections: retrospective observational cohort study. Clin Microbiol Infect. 2020:26(7):897-903.

14. Maskarinec S, Park L, Ruffin F, Turner N, Patel N, Eichenberger E, van Duin D, Lodise T, Fowler V Jr, Thaden JJCM, et al. Positive follow-up blood cultures identify high mortality risk among patients with gram negative bacteremia. Clin Microbiol Infect. 2020;26(7):904-10.

15. Spaziante M, Giuliano S, Ceccarelli G, Alessandri F, Borrazzo C, Russo A, Venditti M. Gram-negative septic thrombosis in critically ill patients: a retrospective case-control study. Int J Infect Dis. 2020;94:110-5.

16. Anderson DR, Morgano GP, Bennett C, Dentali F, Francis CW, Garcia DA, Kahn SR, Rahman M, Rajasekhar A, Rogers FB. American Society of Hematology 2019 guidelines for management of venous thromboembolism: prevention of venous thromboembolism in surgical hospitalized patients. Blood Adv. 2019;3(23):3898-944.

17. Spyropoulos AC, Ageno W, Cohen AT, Gibson CM, Goldhaber SZ, Raskob GJT. Haemostasis: Prevention of Venous Thromboembolism in Hospitalized Medically III Patients: A US Perspective. Thromb Haemost. 2020;120(06):924-36.

\section{Publisher's Note}

Springer Nature remains neutral with regard to jurisdictional claims in published maps and institutional affiliations.

Ready to submit your research? Choose BMC and benefit from:

- fast, convenient online submission

- thorough peer review by experienced researchers in your field

- rapid publication on acceptance

- support for research data, including large and complex data types

- gold Open Access which fosters wider collaboration and increased citations

- maximum visibility for your research: over $100 \mathrm{M}$ website views per year

At $\mathrm{BMC}$, research is always in progress.

Learn more biomedcentral.com/submissions 